\title{
Recent Outpatient Services in General Internal Medicine: $6.4 \%$ of Symptomatic Patients Were Post-Acute COVID-19
}

\author{
Yusuke Hanioka' \\ Ryo Morita ${ }^{2}$ \\ Keiko Yamagami ${ }^{1}$ \\ Shuhei Yao' \\ Ryota Nakamura' \\ Tomoyuki Nakamura' \\ Katsushu Shimizu ${ }^{3}$ \\ Toshimasa Yamaguchi ${ }^{3}$ \\ Hitoshi Goto' \\ 'Department of Internal Medicine, Osaka \\ City General Hospital, Osaka, Japan; \\ ${ }^{2}$ Department of Infectious Diseases, \\ Osaka City General Hospital, Osaka, \\ Japan; ${ }^{3}$ Department of Primary Care and \\ Advanced Triage Section, Osaka City \\ General Hospital, Osaka, Japan
}

Correspondence: Yusuke Hanioka Department of Internal Medicine, Osaka City General Hospital, 2-13-22

Miyakojima-Hondori, Miyakojima-ku,

Osaka, 534-002I, Japan

Tel +8I-6-6929-|22I

Fax +8I-6-6929-204I

Email y-hanioka@med.osakacity-hp.or.jp
Purpose: It is important to understand how COVID-19 is affecting general outpatient services, since hospitals see a wide variety of patients. This study aimed to evaluate the incidence and clinical picture of COVID-19 in general outpatient services. Currently, the diagnosis of COVID-19 depends strongly on the results of polymerase chain reaction (PCR) assays. However, since the sensitivity of PCR tests for SARS-CoV-2 is not high enough to assure confidence. On the other hand, the SARS-CoV-2 antibody (Ab) test is highly sensitive after 2 weeks of symptom onset, and might complement the PCR test. Therefore, we measured $\mathrm{Ab}$ in addition to PCR to obtain a more accurate clinical profile of COVID-19, which might be helpful in building future practice strategies.

Patients: The study patients were those who visited our department for the first time between November 2020 and March 2021 and fulfilled the enrolment criteria.

Methods: All patients underwent total SARS-CoV-2 Ab testing, and PCR tests were performed in only some of them; patients were classified according to the performance of PCR tests for comparisons.

Results: Ninety-four patients who underwent $\mathrm{Ab}$ testing during the study period were eligible for study enrolment, and the PCR test was performed in 40 of them. Only one patient was diagnosed with acute stage COVID-19 based on a positive PCR test. Antibodies were positive in six $(6.4 \%)$ of the 94 patients. Five of the six Ab-positive patients were negative for PCR, and the test was not performed in the sixth patient. All the six patients had prior symptoms suggestive of infection, and respiratory symptoms were more noticeable than fever. The Ab positivity rate was significantly higher than published data.

Conclusion: COVID-19 is not rare in general outpatient services and can be missed based on PCR tests alone. The diagnosis should be made from a comprehensive perspective.

Keywords: COVID-19, SARS-CoV-2 antibody, outpatient service, fever

\section{Introduction}

It has been more than a year since COVID-19 became a global pandemic. This emerging infectious disease has shown no signs of subsiding and is forcing us to restrict our daily lives. In contrast to the early stages of the pandemic, guidelines for SARS-CoV-2 are now being developed, and rapid polymerase chain reaction (PCR) tests for SARS-CoV-2 are now available at many medical institutions. ${ }^{1}$ We provide general medicine outpatient services at our department, and as a result of this, or perhaps because of the needs of society, there has been an increase in the number of patients visiting our department with negative PCR test results, but who continue to 
have fever and other symptoms, and hence, need further evaluation. In the current global pandemic, whether or not a patient is COVID-19 positive is becoming one of the most important factors to be decided in outpatient general medicine. However, we still do not know enough about this new infectious disease. As general physicians, we do not have a complete picture of the impact of COVID-19 on our practice, and there are several clinical issues that need to be addressed, including the safety of hospital staff and others.

Although a PCR assay for COVID-19 has been developed, it cannot completely rule out infection because its sensitivity is $90 \%$ at best, and, unfortunately, there is currently no rapid, objective, and reliable method to differentiate COVID-19., ${ }^{2,3}$ Computed tomography scan of the chest is useful to improve the sensitivity of COVID-19, but it cannot be performed as routine screening due to the problems of radiation exposure and since it does not provide microbiological confirmation. ${ }^{4}$ Hence, although many patients with fever and cough are referred to our outpatient service on a daily basis, there is no satisfactory solution as to how to handle them, as this usually depends on the judgment of the physician in charge.

COVID-19 has been reported to cause sequelae even after recovery from the acute phase. ${ }^{5,6}$ Consequently, patients might visit the clinic for COVID-19-related symptoms that occur after the acute phase has passed. Although this is not limited to COVID-19, diseases triggered by viral infections, such as reactive arthritis, are encountered in daily clinical practice, and it is not rare to be unsure of the differential diagnosis. If a diagnosis of prior viral infection can be made, it might be possible to avoid unnecessary tests and medications; hence, there is clinical benefit in making a diagnosis even if the infection has passed. Given the above clinical scenario and in order to determine the approximate percentage of patients presenting for outpatient medical services who are COVID-19 positive, which would be useful information in clinical practice, we decided to evaluate COVID-19 in first-time outpatients.

Although the situation at the time of writing is different from that at the time the study was conducted because vaccination is now generally available, we have summarized the results because we believe that the clinical characteristics of the patients obtained here might be useful information in future.

\section{Materials and Methods Background of Our Department}

Our hospital is one of the central hospitals in Osaka City and is organized into 58 specialized departments. Our Department of Internal Medicine is akin to a department of general medicine. Therefore, patients who visit our department have a variety of chief complaints, and the diseases we diagnose cover all departments. The main role of our department is to provide medical services to patients who are referred from other hospitals, and many of them visit us in order to find the cause of their symptoms. Since our hospital is a Class 1 designated infectious disease medical institution, we routinely perform medical treatment for COVID-19 patients. However, the Department of Infectious Diseases and the Department of Intensive Care Medicine are mainly responsible for the treatment of patients in the acute phase of COVID-19. In outpatient services, patients with fever of over $38.0^{\circ} \mathrm{C}$ within the previous seven days, patients with respiratory symptoms even with fever of $37.5^{\circ} \mathrm{C}$, and patients returning from overseas are triaged to the Department of Infectious Diseases. Therefore, the probability of acute COVID-19 patients visiting our department is low. However, COVID-19 patients who fall outside this triage might be seen at our department.

\section{Evaluation of COVID-19}

Although COVID-19 is usually assessed by PCR testing when it is deemed appropriate, we mainly used SARS-CoV-2 antibody $(\mathrm{Ab})$ assays at the time of conducting this study for the following reasons. Patients who visit our department have usually had symptoms since a while and have been previously undergoing tests elsewhere. Hence, when they first visit our department, patients might be past the appropriate time for PCR testing, or PCR testing might have already been performed at the referring institution. Consequently, an $\mathrm{Ab}$ test was considered more appropriate for microbiological testing of SARS-CoV-2. Additionally, we chose Ab testing due to the fact that vaccines were not widely available at the time this study was conducted, allowing for simple interpretation of the presence of antibodies. In addition, rheumatoid factor $(\mathrm{RF})$ was measured in SARS-CoV-2 Ab-positive patients, because RF is known to be cross-reactive with SARS-CoV-2 Ab. ${ }^{7}$

\section{Antibody Assay}

The assay reagent used in this study was Elecsys ${ }^{\circledR}$ AntiSARS-CoV-2 from Roche Diagnostics (Indianapolis, IN, USA), which is based on the electrochemiluminescence immunoassay method. This assay reagent detects complex 
antibodies containing immunoglobulin $\mathrm{G}$ targeting the nucleocapsid of SARS-CoV-2, and has a sensitivity of $99.5 \%$ and a specificity of $99.8 \%$ for specimens obtained 2 weeks after onset of infection (Cut off index 1.0). ${ }^{8}$

\section{Study Patients}

From November 1, 2020 to March 31, 2021, we performed usual medical treatment at our Department of Internal Medicine for new patients. Additionally, the presence of COVID-19 was assessed in patients aged 18 years or older who visited our department with any kind of undiagnosed symptoms, and who consented to study participation. Patients who met the following criteria were excluded because they did not meet the purpose of this study: (i) Patients in whom a diagnosis was already or almost confirmed at the time of the first visit, for example, a request for continued medical care for rheumatoid arthritis, or detailed examination of a tumor, (ii)
Patients who visited us for a secondary examination of laboratory abnormalities without symptoms, (iii) Patients who had already received some form of SARS-CoV-2 vaccine or had had COVID-19, and (iv) Patients who were so immunocompromised that they could not be expected to produce globulins, as in X-linked agammaglobulinemia. We also measured SARS-CoV-2 Ab titers in 10 patients who were known to have had COVID-19 in the past as positive controls, and in 26 volunteers who had no particular symptoms as negative controls. All 26 volunteers are doctors at our hospital, including two infectious disease physicians, 12 intensivists and 12 internists who routinely treat COVID-19 patients.

\section{Classification of Chief Complaint}

We summarized the background and clinical features of the patients who were positive for COVID by PCR or antibody testing (Table 1) to examine the clinical

Table I Summary of Patients with Positive Antibody or PCR Tests for SARS-CoV-2

\begin{tabular}{|c|c|c|c|c|c|c|c|c|c|}
\hline No. & Age & Sex & CC & PDay & $\mathbf{A b}$ & (Titer) & PCR & $\mathbf{c X R}$ & CT \\
\hline & \multicolumn{9}{|c|}{ Case description } \\
\hline \multirow[t]{2}{*}{ I } & $39 y$ & $M$ & Fever & 14 & + & 35.6 & - & - & $\mathrm{n} / \mathrm{a}$ \\
\hline & \multicolumn{9}{|c|}{ He had a high-grade fever and cough, which improved by the time of his visit. } \\
\hline \multirow[t]{2}{*}{2} & 39 y & $\mathrm{F}$ & Cough & 61 & + & 15.0 & $\mathrm{n} / \mathrm{a}$ & $\mathrm{n} / \mathrm{a}$ & $\mathrm{n} / \mathrm{a}$ \\
\hline & \multicolumn{9}{|c|}{ She had upper respiratory symptoms about two months earlier, but did not seek medical attention. She was a close contact of a COVID-I9 patient. } \\
\hline \multirow[t]{2}{*}{3} & $22 y$ & $F$ & Fever & 21 & + & 4.2 & - & - & - \\
\hline & \multicolumn{9}{|c|}{$\begin{array}{l}\text { She visited our outpatient service for low-grade fever, fatigue and dry cough. She had high fever } 3 \text { months earlier and } 3 \text { weeks earlier, but the } \\
\text { SARS-CoV-2 PCR test was negative both times. }\end{array}$} \\
\hline \multirow[t]{2}{*}{4} & 54 y & $\mathrm{F}$ & Fatigue & 23 & + & 35.6 & - & + & + \\
\hline & \multicolumn{9}{|c|}{$\begin{array}{l}\text { She experienced headache, fatigue and fever for a day, and although the fever improved, fatigue persisted. Since she was a close contact of } \\
\text { a COVID- } 19 \text { patient, the PCR test was performed twice, but was negative both times. }\end{array}$} \\
\hline \multirow[t]{2}{*}{5} & $56 y$ & $\mathrm{~F}$ & Cough & 19 & + & 12.8 & - & $\mathrm{n} / \mathrm{a}$ & + \\
\hline & \multicolumn{9}{|c|}{$\begin{array}{l}\text { She did not have a fever, but visited us for persistent cough. Since the patient was a close contact of a COVID-I9 patient, PCR tests were } \\
\text { performed twice, but both were negative. }\end{array}$} \\
\hline \multirow[t]{2}{*}{6} & $32 y$ & $M$ & Arthralgia & 26 & + & 10.9 & - & - & $\mathrm{n} / \mathrm{a}$ \\
\hline & \multicolumn{9}{|c|}{$\begin{array}{l}\text { The patient was referred to the hospital because persistent arthritic symptoms appeared in multiple joints after common cold symptoms. } \\
\text { The symptoms were thought to represent reactive arthritis. }\end{array}$} \\
\hline 7 & $87 y$ & $\mathrm{~F}$ & Dyspnea & 7 & - & 0.1 & + & + & + \\
\hline & She & ts & due $t$ & failu & CT & $\mathrm{tcor}$ & ID- & wa & $t$ th \\
\hline
\end{tabular}

Notes: In the upper part of the table, from left to right, the following items are listed: case number, age, sex, chief complaint, days from onset to consultation, presence of SARS-CoV-2 antibody test and its titer, PCR test result, simple chest radiograph findings and simple chest CT findings. If a test was not performed, it is described as n/a. For chest radiographs and CT, "+" was used when there were findings that could explain COVID-19, and "-" was used otherwise. A brief case description is presented in the lower part of the rows for each patient.

Abbreviations: $C C$, chief complaint; Ab, antibody; PDays, number of days after onset; PCR, polymerase chain reaction; cXR, chest X-ray; CT, computed tomography; n/a, not available. 
Table 2 Antibody Response and Chief Complaint in Patients Grouped According to Whether or Not They Underwent PCR Testing

\begin{tabular}{|c|c|c|c|c|}
\hline & \multicolumn{3}{|c|}{ SARS-CoV-2 PCR } & \multirow{2}{*}{ Case } \\
\hline & $\begin{array}{r}\text { Not } \\
\text { Performed }\end{array}$ & Performed & (Positive) & \\
\hline Ab positive* & I (1.9\%) & $5(12.5 \%)$ & 0 & \\
\hline Ab negative* & $53(98.1 \%)$ & 35 (87.5\%) & 1 & \\
\hline Fever** & $23(42.6 \%)$ & 31 (77.5\%) & 0 & No. I, 3 \\
\hline Non-fever** & 31 (57.4\%) & 9 (22.5\%) & 1 & \\
\hline Arthralgia & $6(11.1 \%)$ & I (2.5\%) & 0 & No. 6 \\
\hline Fatigue & $3(5.6 \%)$ & $2(5.0 \%)$ & 0 & No. 4 \\
\hline Dyspnea & $2(3.7 \%)$ & I (2.5\%) & 1 & No. 7 \\
\hline Stomach pain & $3(5.6 \%)$ & $0(0 \%)$ & 0 & \\
\hline Sore throat & $2(3.7 \%)$ & $0(0 \%)$ & 0 & \\
\hline Loss of appetite & I (1.9\%) & I (2.5\%) & 0 & \\
\hline Cough & I (1.9\%) & I (2.5\%) & 0 & No. 2,5 \\
\hline Other & $13(24.1 \%)$ & $3(7.5 \%)$ & 0 & \\
\hline Total & 54 & 40 & I & \\
\hline
\end{tabular}

Notes: Since there was only one case that was PCR positive, it has been shown as an auxiliary column next to the "PCR performed" column. The numbers shown in the "Case" column correspond to the cases shown in Table I. * and **Indicate the items that showed significant differences at $p<0.05$ and $p<0.001$, respectively, between the PCR test performed and not performed groups. All except one of the antibody-positive patients underwent PCR testing; the patient in whom PCR was not performed was not evaluated because he did not visit the hospital in the acute phase. In segregation according to the chief complaint, PCR was performed significantly more often in patients with fever. The complaints other than fever are listed in the order of frequency and importance below "Non-fever". Five Abpositive patients were PCR negative, and the sixth Ab-positive patient did not undergo PCR testing.

Abbreviations: Ab, antibody; PCR, polymerase chain reaction.

characteristics of SARS-CoV-2 patients. In addition, the entire study cohort was divided into two groups, PCR notperformed and PCR performed, based on whether or not they underwent PCR testing, and the antibody status and chief complaint of patients in the two groups were compared (Table 2). In this analysis, we assessed the patients' main complaint, based on the patient's subjective complaints, regardless of the description in their referral letter.

\section{Statistical Analysis}

We performed a $\chi^{2}$-square test to compare the two groups for each item in Table 2. Probability values of less than 0.05 were considered statistically significant.

\section{Results}

During the study period, 275 patients were seen for the first time at our department, among whom 94 consenting patients who met the inclusion criteria were enrolled in this study. Among these patients, 40 patients were also examined using SARS-CoV-2 PCR tests within thirteen days of symptom onset; 31 of the 40 patients presented with fever as the main complaint and 9 patients presented with other complaints. Only one patient without fever was PCR test-positive for COVID-19 at the time of the first visit to our department, while all the other 39 patients were negative. This PCR-positive case was diagnosed as acute stage COVID-19 on the basis of PCR and radiography results. Since this patient died 11 days later, Ab levels in serum on only day 7 of illness were assessed, while in all the other 93 patients, Ab levels were measured 2 weeks or more after symptom onset. There were six SARS-CoV-2 Ab-positive patients, two with fever and four without fever, all of whom had a history suggestive of some prior infection. SARS-CoV-2 PCR testing had been performed in the acute phase in five of the six patients and was negative as described above, while the PCR test was not performed in one patient because she did not seek medical attention at that time. In addition, all six of these patients had RF levels below the limit of detection. A summary of the seven patients who tested positive for SARS-CoV-2 by PCR (1 patient) or Ab testing (6 patients) is shown in Table 1. Additionally, a summary of the $\mathrm{Ab}$ titers and the patients' chief complaints categorized by whether or not PCR testing was performed is shown in Table 2. All 10 positive controls were positive for SARS$\mathrm{CoV}-2 \mathrm{Ab}$, and all 26 volunteers were negative.

\section{Discussion}

Although the PCR test for SARS-CoV-2 provides rapid results and has a high positive predictive value, it is not foolproof and should not be completely depended on in COVID-19 practice. The sensitivity of the PCR test is generally considered to be around $70 \%$, or $90 \%$ at the highest. ${ }^{3,8}$ In addition, patients are not always seen at the right time, and the accuracy of the result depends on how well the procedure is performed. In fact, there are several reports of COVID-19 cases where the initial PCR test was negative. $^{9-12}$ The Ab test only requires a blood sample, so the results are less likely to be influenced by individual factors. Abs are detectable in serum from about 7 days after infection onset and persist for at least 3-6 months. ${ }^{8,13,14}$ The assay reagent used in this study provided a very high sensitivity and specificity after two weeks of onset, as mentioned above. ${ }^{7}$ Thus, the diagnostic accuracy is considered to be very high in cases with a history of suspected infection. This is also mentioned in the Infectious Disease Society of America guidelines, which indicate that $\mathrm{Ab}$ testing should be considered in 
patients with a strong clinical suspicion of COVID-19 and negative PCR results. ${ }^{15}$ In the six patients who were $\mathrm{Ab}$ positive in this study, all had symptoms of infection, and more than two weeks had passed between symptom onset and their visit to our department. Additionally, they were negative for RF, suggesting that they were truly COVID19 positive.

In our study, PCR testing was performed with an intense focus on fever. Thirty-one of the 40 patients (77.5\%) who underwent PCR testing were febrile, and even in non-febrile patients, four patients underwent PCR testing because they were close contacts of COVID-19 patients, and one patient had fever in the early stage of the clinical course. Of the six antibody-positive patients, one did not visit the hospital in the acute stage of their illness, and the remaining five cases were patients who underwent the PCR test, indicating that the decision to apply PCR testing was not incorrect. However, since five of the six Ab positive cases had negative PCR test results (the test was not performed in the sixth $\mathrm{Ab}$ positive case) despite having had the infection, it suggests that 5/39 cases $(12.8 \%)$ would have been missed if only PCR testing had been performed. In these cases, fever was observed only in cases No. 1 and 3 at the time of the initial visit, and cases No. 2 and 5 showed no fever throughout the course of the disease. On the other hand, five of the six patients had a history of some kind of respiratory symptoms. In actuality, fever is not an inevitable symptom of COVID-19, and respiratory symptoms, such as cough, are as common as fever. ${ }^{16}$ Therefore, it is necessary to pay attention for symptoms of the common cold as well as fever when considering COVID-19. Since the infectiousness of this disease lasts for up to 9 days after the onset of symptoms, it is not necessary to worry about infectivity in most cases in outpatient services of higher medical institutions, such as our department. ${ }^{17}$ However, even after this period, symptoms related to COVID-19 are still possible and are expected to increase with the spread of COVID-19 in the future, so general physicians should always consider this as a differential diagnosis regardless of the results of PCR testing.

In our study, the positive rate of SARS-CoV-2 Ab was higher than expected. According to a survey by the Ministry of Health, Labour and Welfare of Japan, the SARS-CoV-2 $\mathrm{Ab}$ positivity rate in the general population in Osaka Prefecture, which was assessed using the same assay, was 10 of 2970 people $(0.34 \%)$ in June 2020 and 25 of 2746 (0.91\%) people in December 2020. ${ }^{18,19}$ Furthermore, as of March 31, 2021, the cumulative number of COVID-19 patients in Osaka Prefecture was 52,202, indicating that the estimated rate of infection based on the population of Osaka Prefecture (8.823 million) was $0.63 \%{ }^{20}$ In comparison, the antibody positivity rate in first-time outpatients at our department was $6.4 \%$, which is significantly higher $\left(p<0.05, \chi^{2}-\right.$ square test). Since the patients we assessed in this study were selected from those for whom the cause of the disease could not be identified even after examination at the primary medical institution, it is not surprising that the number of $\mathrm{Ab}$ positive patients was high. However, from a different perspective, it indicates that many COVID-19 patients are not diagnosed in the acute stage and are subsequently seen at the general outpatient clinic. In addition, when the parameter was limited to only those who were diagnosed with viral infections, 35.3\% (6/17) of patients tested positive for SARSCoV-2 Ab. Our results suggest that from the perspective of a general physician, the probability of a clinician encountering COVID-19 cases is higher than the publicly announced values suggest, and is already at the level that it can be described a common disease.

As described above, COVID-19 is a disease that can be encountered on a daily basis, including for its related symptoms. However, now that vaccination has become more widespread, the current situation might be different from that prevalent at the time when this study was conducted, although vaccine coverage is still not very high in the general population. Since there are still many unvaccinated patients, SARS-COV-2 Ab assessment might continue to provide useful information, and hence, its measurement should be considered based on the patient's medical and vaccination history. In addition, even in vaccinated individuals, there is insufficient confirmation of actual clinical performance of the vaccine, and some immunocompromised patients, especially those with $\mathrm{CD} 8+\mathrm{T}$ cell function abnormalities, have been reported to be infected with COVID-19 and re-infected after seroconversion. ${ }^{21,22}$ Therefore, if the patient is within 9 days of symptom onset, even those who are SARS-CoV-2 Ab and PCR negative, we should be very careful not to disregard their symptoms as just a common cold. COVID-19 is still an unknown entity, and is, therefore, unexpectedly difficult to diagnose with certainty. General physicians need to be aware that they might encounter COVID-19 at any time and pay attention to appropriate infection control.

\section{Limitations}

As described previously, there is a selection bias among patients who visit our department. The probability of diagnosing COVID-19 related symptoms in many medical 
institutions might be lower than in this study. For the same reason, we also had limited opportunities to diagnose COVID-19 in the acute phase, and it is possible that other medical institutions might have more opportunities to diagnose acute COVID-19. Additionally, as vaccination becomes more widespread, the results might be different.

\section{Conclusion}

COVID-19 is currently a widespread infection, and many cases are missed despite performance of the necessary diagnostic tests. Diagnosis and risk management of COVID-19 should be performed comprehensively with reference to the results of medical interviews, microbiological tests, and imaging studies.

\section{Ethics Approval}

This study was approved by the Clinical Research Ethics Board of Osaka City General Hospital (No. 2010114) and was conducted in accordance with the Declaration of Helsinki. All patients and volunteers provided verbal and written informed consent for study inclusion.

\section{Disclosure}

The authors report no conflicts of interest related to this work.

\section{References}

1. Ministry of Health, Labor and Welfare. Clinical management of patients with COVID-19: a guide for front-line healthcare workers Ver 5.2. Available from: https://www.mhlw.go.jp/content/000815065. pdf. Accessed September 302021.

2. Fang Y, Zang H, Xie J, et al. Sensitivity of chest CT for COVID-19: comparison to RT-PCR. Radiology. 2020;298(2):E115-E117. doi:10. 1148/radiol.2020200432

3. Yokota I, Shane PY, Okada Y, et al. Mass screening of asymptomatic persons for SARS-CoV-2 using saliva. Clin Infect Dis. 2020. doi:10. 1093/cid/ciaa1388

4. Ai T, Yang Z, Hou H, et al. Correlation of chest CT and RT-PCR testing for Coronavirus disease 2019 (COVID-19) in China: a report of 1014 cases. Radiology. 2020;296(2):E32-E40. doi:10.1148/radiol.20 20200642

5. Carfi A, Bernabei R, Landi F. Persistent symptoms in patients after acute COVID-19. JAMA. 2020;324(6):603-605. doi:10.1001/jama.20 20.12603

6. Tenforde MW, Kim SS, Lindsell CJ. Symptom duration and risk factors for delayed return to usual health among outpatients with COVID-19 in a multistate health care systems network - United States, March-June 2020. MMWR Morb Mortal Wkly Rep. 2020;69 (30):993-998. doi:10.15585/mmwr.mm6930e1
7. Bausa MV, Peris RB, Corominas H. Cross-reactions between rheumatoid factor and IgM SARS-CoV-2. Med Clin. 2020;155 (9):417-418. doi:10.1016/j.medcli.2020.07.008

8. Lou B, Li TB, Zheng SF, et al. Serology characteristics of SARS-CoV-2 infection since exposure and post symptom onset. Eur Respir J. 2020;56(2):2000763. doi:10.1183/13993003.00763-2020

9. Perez-Garcia F, Perez-Tanoira R, Romanyk J, Arroyo T, GomezHerruz P, Cuadros-Gonzalez J. Alltest rapid lateral flow immunoassays is reliable in diagnosing SARS-CoV-2 infection from 14 days after symptom onset: a prospective single-center study. J Clin Virol. 2020;129:104473. doi:10.1016/j.jcv.2020.104473

10. Guo L, Ren L, Yang S, et al. Profiling early humoral response to diagnose novel Coronavirus disease (COVID-19). Clin Infect Dis. 2020;21:778-785.

11. Liu L, Liu W, Zheng Y, et al. A preliminary study on serological assay for severe acute respiratory syndrome coronavirus 2 (SARS-CoV-2) in 238 admitted hospital patients. Microbes Infect. 2020;22(4):206-211. doi:10.1016/j.micinf.2020.05.008

12. Pan Y, Li X, Yang G, et al. Serological immunochromatographic approach in diagnosis with SARS-CoV-2 infected COVID-19 patients. $J$ Infect. 2020;81(1):e28-e32. doi:10.1016/j.jinf.2020.03.051

13. Yamayoshi $\mathrm{S}$, Yasuhara $\mathrm{A}$, Ito $\mathrm{M}$, et al. Antibody titers against SARS-CoV-2 decline, but do not disappear for several months. EClinicalMedicine. 2021;32:100734. doi:10.1016/j.eclinm.2021.100 734

14. Long QX, Lui BZ, Deng H, et al. Antibody responses to SARS-CoV2 in patients with COVID-19. Nat Med. 2020;26(6):845-848. doi:10. 1038/s41591-020-0897-1

15. Hanson KE, Caliendo AM, Arias CA, et al. Infectious Diseases Society of America Guidelines on the diagnosis of COVID-19: serologic testing. Clin Infect Dis. 2020. doi:10.1093/cid/ciaa1343

16. Stokes EK, Zambrano LD, Anderson KN, et al. Coronavirus disease 2019 case surveillance - United States, January 22-May 30, 2020. MWR Morb Mortal Wkly Rep. 2020;69(24):759-765. doi:10.15585/ mmwr.mm6924e2

17. Cevik M, Tate M, Lloyd O, Maraolo AE, Schafers J, Ho A. SARSCoV-2, SARS-CoV, and MERS-CoV viral load dynamics, duration of viral shedding, and infectiousness: a systematic review and meta-analysis. Lancet Microbe. 2021;2(1):e13-22. doi:10.1016/ S2666-5247(20)30172-5

18. Ministry of Health, Labor and Welfare in Japan. Available from: https://www.mhlw.go.jp/stf/newpage_11892.html. Accessed June 16, 2020.

19. Ministry of Health, Labor and Welfare in Japan. Available from: https://www.mhlw.go.jp/content/000734482.pdf. Accessed February 5, 2021.

20. Osaka Prefectural Government in Japan. Available from: https://pref. Osaka.lg.jp. Accessed June 30, 2021.

21. Bentivegna E, Sentimentale A, Luciani M, Speranza ML, Guerritore L, Martelletti P. New IgM seroconversion and positive RT-PCR test after exposure to the virus in recovered COVID-19 patient. J Med Virol. 2021;93(1):97-98. doi:10.1002/ jmv. 26160

22. Luciani M, Bentivegna E, Spuntarelli V, et al. Recurrent COVID-19 pneumonia in the course of chemotherapy: consequence of a weakened immune system? J Med Virol. 2021;93(4):1882-1884. doi:10.1002/jmv.26701 


\section{Publish your work in this journal}

The International Journal of General Medicine is an international, peer-reviewed open-access journal that focuses on general and internal medicine, pathogenesis, epidemiology, diagnosis, monitoring and treatment protocols. The journal is characterized by the rapid reporting of reviews, original research and clinical studies across all disease areas. The manuscript management system is completely online and includes a very quick and fair peer-review system, which is all easy to use. Visit http://www.dovepress.com/ testimonials.php to read real quotes from published authors. 